\title{
Tentative Analysis of the Function of Applying Computer Database Technology in Information Management
}

\author{
Xie Peng \\ Yunnan College of Business Management, Anning, 650106, China
}

Keywords: Information management ,Computer, Database technology

\begin{abstract}
With the development of information technology and the progress of science and technology, information management has been widely used in computer data technology, and continuously improve the level of information management .This paper mainly analyzes the application status of computer database technology in information management, application features and puts forward the application of information management computer database technology measures for the further effective application of information resources and information management.
\end{abstract}

\section{Introduction}

In the information age, the daily work and communication will form a large amount of data, in the process of dealing with the above data, it should be reasonable to use the corresponding computer database technology. In the process of information management, the application of computer database technology can ensure to managing information efficiently and accurately . In the 1960' $\mathrm{s}$ emerged the database technology, the main function was to store data resources and to carry out effective management. At present, the database technology has been gradually integrated into the computer system. People pay more and more attention to the database technology. But what the database technology is, what the characteristics of the database technology are, how it is applied to the information management, we will analyze and discuss the above aspects in this paper..

\section{The present situation of application of computer database technology in information management}

\section{High speed development}

High speed development of computer technology will promote and develop rapidly the computer database technology.We can not ignore the role and significance of the application of computer database technology in information management, resulting in more and more professional staff focus on the development and research of database technology. The use of computer database technology is closely related to management information quality,that' $s$ why we should develop rapidly the computer database technology to some extent and put it into the information management reasonable,which can provide foundation for the of database technology.

\section{An increasingly wide range of applications}

Computer database technology has been widely used in the application of computer technology, which can guarantee the high speed development. Because of the increasing market demand, it has more and more application of computer database, in the development of the third industry, agriculture, industry leader, the use of computer database system to manage information has become an essential tool for industry development.

\section{Strengthening the reliability}

The basic guarantee of running computer database system is information security,which can avoid the problem of tampering information and leakage information.Due to the rapid development of computer technology, in the process of information management, information security privacy issues become an urgent need to solve the problem, and continuously improve the database security capabilities can ensure that management information has a higher security,which can improve the overall security of the database. 


\section{Increasing attention}

Continuous development and improvement of computer technology has brought great convenience to people's daily life, computer database technology is applied in various fields . So, more enterprises begin to take computer database technology as management information auxiliary tools to increase the work efficiency .At present, a lot of work can not leave computer database technology, for example such as processing data, electronic commerce and other [1].

\section{The characteristics of the application of computer database system in information management}

\section{Better sharing performance}

The most important feature of the computer database system is the information sharing, in the beginning of the establishment of the database, mainly in order to achieve the purpose of a total of information. The database system is built on the basis of the whole system. Therefore, the user and the multiple programs simultaneously use the database system to share data, and can get more storage space, so as to achieve the purpose of compatible data, it is an important way to carry out data management.

\section{Good data regularity}

The computer system is scattered disorder data, even after the regular processing of data,it can not effectively contact the relevant data, the database system can be a reasonable integration, the establishment of a certain degree of contact with the database, in order to greatly improve the level of data.

\section{Better data independent performance}

Data independent performance is said to be two aspects, data logic independence and physical independence. The independence of data logic is that the application and the logic structure of the database data system are independent of each other. It is the time to change the data type and the definition. It is not to change the original program. The data physical independence is not because the structure of the storage data changes the logical structure of the database.

\section{Higher data reliability}

With the rapid development of computer technology, the overall security of the database system has been improved, and the strength of management data has been continuously increased.

\section{Higher data flexibility}

Management of the database of the time there is a general process of processing data, not just a simple accumulation of data, but with a certain management functions. You can complete the conversion between the database, select the database to ensure a more efficient database system, in order to reduce the cost of management information, in addition, it should be constantly optimize the allocation of information resources.

\section{Unified control and optimization of management data}

The most important problem in computer network operation is data sharing and management. In addition, it is also the most favorable advantage of the database system itself. Database can be used to handle multiple users, a database is not only in a department or enterprise sharing, can be in different countries, different regions, different business users to share, so as to form a comprehensive data network.

\section{Data structuralization}

Compared with the ottems ,the Computer database system is different. It can effectively solve the problem of the files are not relevance in the system, in order to achieve the purpose of the structure of the overall data.The same data has a certain connection and organization structure, the whole body should follow the corresponding structural rules and principles, as well as the same data characteristics, but also the difference between file system and database system based on [2].

\section{Controllable redundancy of database}

Database redundancy is mainly the repeated data in the database. Database has a certain grasp. Because of its high frequency of use, the general situation will not form data redundancy for the purpose of sharing data In the design of database systems, the need to eliminate redundant data, query 
database, to ensure that a certain query rate, processing duplicate data, to ensure reasonable control redundancy.

\section{The use of computer database technology in information management}

In the process of information management, computer database technology has very obvious effect, and also continue to expand the scope of application of computer database system. Because the computer database technology is a network technology,and the existence of the current network complex environment and the rapid development of network technology, which makes the application of computer database system some problems.At this stage, the data information network security is the biggest hidden dangers exist.How to protect the overall security of data information, to avoid malicious information, steal information, destruction of information, is an important problem in information management. It is still a big challenge in the actual use of database, if not deal with the above the problem in time, there will be serious consequences for the information management, and cause irreparable, immeasurable loss. So, in the analysis and research of computer database system, the applicability and safety guarantee system, can be analyzed from the following aspects:

\section{Improving access management technology}

There are two aspects of the main contents of the access management technology in the database system, access control technology and user authentication technology. User identification is the main means that the computer database system to provide users with a technology that can identify their own identity, the use of authorized form of database can determine the user, once the user needs access to the system, the system will automatically identify and authenticate user identity, the release is legitimate users. At this stage, the use of password to verify the user's identity has a relatively high security. With the development of technology, the need to continue to develop new technologies, for example, iris authentication technology, fingerprint authentication technology. Access control technology is the computer database system can access control access to the system of users, can further protect information security, according to the user's actual level of reasonable access, improve the management database, the general situation is the use of RBAC in the role of access control.

\section{Strengthen the backup and recovery technology}

The main way to ensure the safe operation of computer database system is data recovery and data backup technology. Data backup means that the relevant personnel copy and save the database content, that is, the integration of existing normal data, to avoid damage or loss of data problems, the general situation of the use of the backup data is a dynamic backup and static backup, and effectively grasp the backup work, to protect the maximum safe operating database system. Data recovery is the recovery of the relevant data to the problem before the situation, it is necessary to backup the system ahead of the relevant data, to ensure that the system information was damaged. The recovery system data has a certain treasure, it is necessary to continuously improve data recovery and backup technology to ensure system security.

\section{Enhanced intrusion detection technology}

Intrusion detection technology is to set up multiple levels and authentication information to run the database system to ensure the security of the system as a whole data information. If network attack or virus attack, the above technical means can be effective, timely and quickly find malicious behavior, and then take protective measures, for example, network connection cut off, issued a warning signal, to ensure the normal operation of the database system, and gradually become an important protection system. But at present the technology is not very perfect, which need to improve technology to ensure that a more secure operating environment [3].

\section{The strategy of application of computer database technology in information management}

Application of computer database technology in information management has become a trend in the future, with the continuous development of database technology, has been a certain effect, but there are some problems and defects, so, need to strengthen the effective use of computer database 
technology, increasing the overall level of management information, in order to take effective measures.

\section{To be more connected with practice and theory in application}

Because of the application of computer database technology in different fields, and in the process of practice, we need to establish the latest research database technology, the theory of the development of the theory can promote the practice, and the development of the new theory.

\section{Continuously improve the security of computer database system}

Along with the continuous development and updating of computer database technology, units and enterprises are also more and more applications and the introduction of computer database technology, business unit information management application of computer database technology, the need to focus on security issues, in the continuous strengthening of the database technology as a whole, can ensure that the database will not be malicious tampering and illegal use. Although the shared database system can bring great convenience to people's daily life, but also can be a security problem, therefore, in order to ensure that the database system has a certain share of time, also need to take into account the confidentiality of the military, national interests, business secrets and other data information security, and use effective solutions to improve the overall security of the data system.

\section{The strengthening of the comprehensive data}

The most basic requirements of running the database data technical data integrity, data integrity will greatly affect the level of information management and the effect of the application of database technology, therefore, the need to effectively use the database technology, it is required to ensure that a complete database system data [4].

Improving the ability of the database system.

At present, people's life and work environment, there will be a large variety of information, how effective, reasonable use of the surrounding information has gradually become an important problem, will reduce the overall working efficiency. Therefore, it is necessary to continue to integrate the techniques of computer and database information capacity, maximize reduce repeat redundant information, so it can be more convenient to management information system, to ensure that users can quickly get the corresponding information, to maximize the value of information, provide guarantee and basis for the further development of computer database system, improve the management efficiency of information [5].

\section{Concluding remarks}

In a word, it is very important to find the database technology in the information management after the full understanding of the technology of the computer database. In the information age, information management is becoming more and more important. The computer system is an important part of the database technology, and gradually in the management of information management features, and more emphasis on information management, due to the extensive application of computer technology, the need to continue to study and develop new database technology, and promote the use of database technology, social and corporate sustainable development.

\section{References}

[1] Xiao Huiqiang. Study on the application of computer database in information management. computer software and application, 2014 (8): 146-146148.

[2] Huang Changwei. Study on the application of computer database in information management. wireless Internet technology, 2014 (10): 87-87.

[3] Zhu Minghui. Analysis of the application and the development direction of computer database in information management. Curriculum education research (new teachers teaching), 2014 (8): 283-283. 
[4] Wang Caixia. Study on the application of computer database in information management. wireless Internet technology, 2014 (4): 191-191.

[5] Xiong Jing. The application of computer database used in Information management. Information and Computer, 2015 (6): 89-90. 